Med Klin Intensivmed Notfmed 2020 · 115:529 https://doi.org/10.1007/s00063-020-00742-8

(c) Springer Medizin Verlag GmbH, ein Teil von Springer Nature 2020

\author{
M. Buerke ${ }^{1,2} \cdot$ C. Hermes ${ }^{3} \cdot$ S. Petros ${ }^{4,5}$ \\ ${ }^{1}$ Herz und Gefäßzentrum Südwestfalen, Klinik für Innere Medizin II, Kardiologie, Angiologie, Internistische \\ Intensivmedizin, St. Marien-Krankenhaus Siegen, Siegen, Deutschland \\ ${ }^{2}$ Universitätsklinik für Innere Medizin III, Martin-Luther-Universität Halle-Wittenberg, Halle/Saale, \\ Deutschland \\ ${ }^{3}$ Bonn, Deutschland \\ ${ }^{4}$ Interdisziplinäre Internistische Intensivmedizin, Universitätsklinikum Leipzig, Leipzig, Deutschland \\ ${ }^{5}$ Medizinische Klinik 1, Bereich Hämostaseologie, Universitätsklinikum Leipzig, Leipzig, Deutschland
}

\title{
Klinische Skills
}

Laborbefunden und klinischen Daten können oft unentdeckt bleiben, weil Einige nicht über die entsprechenden Fähigkeiten verfügen, die klinischen Daten in Kombination mit den Befunden zu analysieren. Oft werden offensichtlich unlogische Laborbefunde ohne Frage akzeptiert. Eine angemessene Anamnese und körperliche Untersuchung sind zwar für die Ermittlung der richtigen Diagnose unerlässlich, aber ohne die Fähigkeit, gesammelte Informationen zu interpretieren, nicht zielführend. Häufig werden die Anamnese durch geschlossene Fragen eingeschränkt bzw. spezifische Details nicht erfasst, sodass die klinische Entscheidungsfindung eingeschränkt ist. Des Weiteren ist eine mangelnde interprofessionelle Kommunikation und Informationsweitergabe zu beobachten. Zur Verbesserung der Ausbildung klinischer Fähigkeiten, auch in der Facharztausbildung und pflegerischen Weiterbildungen im Krankenhaus, sind diese Aspekte wesentlich und längst überfällig. In Anerkennung dessen, dass die Bereitstellung hochwertiger medizinischer Versorgung auf der Intensivstation klinische Fähigkeiten und klinisches Denken erfordert, hat die Deutsche Gesellschaft für Intensiv- und Notfallmedizin ein Curriculum für Intensiv- und Notfallmedizin erstellt, um die ärztliche Aus- und Weiterbildung langfristig $\mathrm{zu}$ verbessern. Mit diesem Heft wollen wir einen weiteren Beitrag leisten, um wichtige klinische Skills zu thematisieren.

Wir wünschen Ihnen viel Freude beim Lesen.

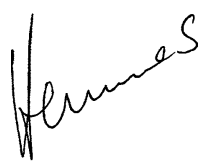

Carsten Hermes

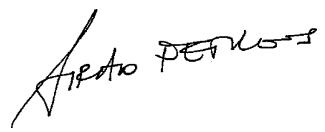

Prof. Dr. med. Sirak Petros

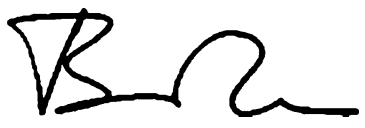

Prof. Dr. med. Michael Buerke

\section{Korrespondenzadresse}

Prof. Dr. med. M. Buerke

Herz und Gefäßzentrum Südwestfalen, Klinik für Innere Medizin II, Kardiologie, Angiologie, Internistische Intensivmedizin, St. MarienKrankenhaus Siegen

Kampenstr. 51, 57072 Siegen, Deutschland m.buerke@mariengesellschaft.de

Interessenkonflikt. M. Buerke, C. Hermes und S. Petros geben an, dass kein Interessenkonflikt besteht. 